\title{
Adaptive Synchronization of Complex Dynamical Networks Governed by Local Lipschitz Nonlinearlity on Switching Topology
}

\author{
Bo Liu, ${ }^{1}$ Xiaoling Wang, ${ }^{2}$ Yanping Gao, ${ }^{3}$ Guangming Xie, ${ }^{4}$ and Housheng Su \\ ${ }^{1}$ College of Science, North China University of Technology, Beijing 100144, China \\ ${ }^{2}$ Department of Automation, Shanghai Jiao Tong University, Shanghai 200240, China \\ ${ }^{3}$ College of Computer and Information Engineering, Beijing Technology and Business University, Beijing 100048, China \\ ${ }^{4}$ Department of Industrial Engineering and Management, College of Engineering, Peking University, Beijing 100871, China \\ ${ }^{5}$ Department of Control Science and Engineering, Key Laboratory of Image Processing and Intelligent Control of Education Ministry \\ of China, Huazhong University of Science and Technology, Wuhan 430074, China
}

Correspondence should be addressed to Guangming Xie; xiegming@pku.edu.cn

Received 20 December 2012; Accepted 12 August 2013

Academic Editor: Yannick De Decker

Copyright (C) 2013 Bo Liu et al. This is an open access article distributed under the Creative Commons Attribution License, which permits unrestricted use, distribution, and reproduction in any medium, provided the original work is properly cited.

This paper investigates the adaptive synchronization of complex dynamical networks satisfying the local Lipschitz condition with switching topology. Based on differential inclusion and nonsmooth analysis, it is proved that all nodes can converge to the synchronous state, even though only one node is informed by the synchronous state via introducing decentralized adaptive strategies to the coupling strengths and feedback gains. Finally, some numerical simulations are worked out to illustrate the analytical results.

\section{Introduction}

In recent years, the research on the synchronization of complex dynamical networks has attracted great attention and emerged a large number of outstanding works (e.g., [1-8] and the references therein).

For a network, the synchronization implies that all nodes will converge to the same state, which can be a homogeneous equilibrium point or a periodic orbit. To enhance the synchronization of such network, a lot of research methods are developed, and one of the most significant methods is to design effective adaptive strategies for the relevant parameters, such as the coupling strengths and the feedback gains $[1-4,8-10]$. In references $[4,10,11]$, there must have information channel by using a special indicator function; however, in reality, the information channel between any two nodes of a network may be lost or changed. Driven by it, we will investigate the synchronization of the complex dynamical networks with switching topology, which can lead to some information channels that occurred as well as another information channels that disappeared, by introducing adaptive strategies to the coupling strengths and feedback gains. Different from [8], the coupling strengths $c_{i j}^{\sigma(t)}(t)$ are dynamic and variable, which can change according to the switching signal, the neighbor rule, and the switched coupling configuration $a_{i j}^{\sigma(t)}$. Yet, "fast switching" [5] is hardly to be realized and bears new features and difficulties. Here, we attack the problem by invoking the theory of differential inclusion and nonsmooth analysis [12-18].

So far, most attentions have been focused on nonlinear dynamics satisfying the globally Lipschitz condition. However, many known systems only satisfy the local Lipschitz condition, such as Lorenz system, Chen system, and FitzHugh-Rinzel system [9-11]. In this paper, we will also examine nonlinear dynamics of such complex network satisfying the local Lipschitz condition.

The main contribution of the current work lies in characterizing synchronization of complex dynamical networks. First, the nonlinear dynamics of all nodes and the 
synchronous goal satisfies the local Lipschitz condition. Second, the adaptive strategies are introduced to the coupling strengths and the feedback gains. Third, the topology of complex dynamical networks can switch even though the information is discontinue, we can also solves the synchronization based on differential inclusion and nonsmooth analysis. In this paper, we will prove that all nodes of the complex dynamical network which is steered by the adaptive strategies can converge to the synchronous goal, even though only one node is informed by the synchronous goal if the neighboring graph remains connected.

This paper is organized as follows. Section 2 describes the model with the nonlinear dynamics satisfying the local Lipschitz condition, and some preliminaries about the nonsmooth analysis and the local Lipschitz condition are given. The main results are shown in Section 3, while Section 4 presents some simulations to illustrate our theoretical results. The conclusion is given in Section 5 .

\section{Preliminaries and Model Formulation}

2.1. Model Formulation. Consider a dynamical network with $N$ nodes, and each node updates its state by

$$
\begin{array}{r}
\dot{x}_{i}(t)=f\left(x_{i}(t)\right)+\sum_{j \in N_{i}(t)} a_{i j}^{\sigma(t)} c_{i j}^{\sigma(t)}(t)\left(x_{j}(t)-x_{i}(t)\right)+u_{i}(t), \\
i=1, \ldots, N,
\end{array}
$$

where $x_{i}(t)=\left(x_{i 1}(t), x_{i 2}(t), \ldots, x_{i n}(t)\right)^{T} \in R^{n}(i=1,2, \ldots, N)$ is the state vector of node $i, t \in[0,+\infty), f: R^{n} \rightarrow R^{n}$ is a continuous map, and $N_{i}(t)$ is the neighboring set of node $i$ at the instant $t$. Notice that the coupling between the nodes may vary in time. Therefore, the interconnection graph $\mathscr{G}_{\sigma(t)}$ of the model is time varying, where $\sigma(t):[0,+\infty) \rightarrow$ $M=\{1,2, \ldots, m\}$ is the switching signal. For all $k \in M$, $A_{k}=\left(a_{i j}^{k}\right) \in R^{N \times N}$ is the weighted coupling configuration of network (1), which has $a_{i i}^{k}=-\sum_{j \in N_{i}(t)} a_{i j}^{k}$. If there exists communication channel between node $i$ and node $j$ at the switching segment $k$, then $a_{i j}^{k}=a_{j i}^{k}>0$; otherwise, $a_{i j}^{k}=0$. $c_{i j}^{\sigma(t)}(t)$ denotes the coupling strengths between node $i$ and node $j . u_{i}(t)$ is the feedback controller designed as

$$
u_{i}(t)=-h_{i} c_{i}(t)\left(x_{i}(t)-x_{0}(t)\right), \quad i=1, \ldots, N,
$$

where $h_{i}=1$, if the node $i$ is controlled; otherwise, $h_{i}=0$; $c_{i}(t)$ is the feedback gain. $\bar{x}(t)$ is a desired synchronous state for network (1) with

$$
\dot{x}_{0}(t)=f\left(x_{0}(t)\right) .
$$

The adaptive strategies on the coupling strengths and the feedback gains are designed as

$$
\begin{gathered}
\dot{c}_{i j}^{\sigma(t)}(t)=k_{i j} a_{i j}^{\sigma(t)}\left(x_{i}(t)-x_{j}(t)\right)^{T}\left(x_{i}(t)-x_{j}(t)\right), \\
\dot{c}_{i}(t)=k_{i} h_{i}\left(x_{i}(t)-x_{0}(t)\right)^{T}\left(x_{i}(t)-x_{0}(t)\right),
\end{gathered}
$$

where $c_{i j}(0) \geq 0, c_{i}(0) \geq 0, k_{i j}>0$, and $k_{i}>0$ are the adaptive parameters of the coupling strength and the feedback gains, respectively.

In the $k$ th (for all $k \in M$ ) time period, the weighted coupling configuration matrix of network (1) is defined as

$$
B_{k}=\left[\begin{array}{cccc}
a_{11}^{k} c_{11}^{k} & a_{12}^{k} c_{12}^{k} & \cdots & a_{1 N}^{k} c_{1 N}^{k} \\
a_{21}^{k} c_{21}^{k} & a_{22}^{k} c_{22}^{k} & \cdots & a_{2 N}^{k} c_{2 N}^{k} \\
\vdots & \vdots & \ddots & \vdots \\
a_{N 1}^{k} c_{N 1}^{k} & a_{N 2}^{k} c_{N 2}^{k} & \cdots & a_{N N}^{k} c_{N N}^{k}
\end{array}\right] \in R^{N \times N},
$$

with $a_{i i}^{k} c_{i i}^{k}=-\sum_{j=1, j \neq i}^{N} a_{i j}^{k} c_{i j}^{k}$.

2.2. Mathematical Preliminaries. In this section, we introduce some useful concepts, assumptions, and lemmas.

\subsubsection{Concepts of Nonsmooth Analysis}

Definition 1 (see [16], Filippov). A vector function $x(\cdot)$ is called a solution of equation $\dot{x}=f(x, t)$ on $\left[t_{0}, t_{1}\right]$ if $x(\cdot)$ is absolutely continuous on $\left[t_{0}, t_{1}\right]$, and for almost all $t \in\left[t_{0}, t_{1}\right]$,

$$
\begin{aligned}
\dot{x} \in K[f](x, t) & \equiv K[f](x) \\
& \triangleq \overline{c o}\left\{\lim f\left(x_{i}\right) \mid x_{i} \longrightarrow x, x_{i} \notin N_{f} \cup N\right\},
\end{aligned}
$$

where $N_{f} \subset R^{n}, \mu\left(N_{f}\right)=0 ; N \subset R^{n}, \mu(N)=0$.

Definition 2 (see [16], generalized directional derivative). The generalized directional derivative of $f$ at $x$ in the direction $v$, denoted by $f^{\circ}(x ; v)$, is defined as

$$
f^{\circ}(x ; v):=\lim _{y \rightarrow x,} \sup _{t \downarrow 0} \frac{f(y+t v)-f(y)}{t},
$$

where $y$ is a vector in the Banach space $X$ and $t$ is a positive scalar.

Definition 3 (see [18]). $f(x): R^{m} \times R \rightarrow R$ is called regular if

(i) for all $v$, the usual one sided directed derivative $f^{\prime}(x ; v)$ exists,

(ii) for all $v, f^{\prime}(x ; v)=f^{\circ}(x ; v)$.

\subsubsection{Assumptions}

Assumption 1. If each $f_{i}$ of the nonlinear function $f\left(x_{i}(t)\right)=$ $\left(f_{1}\left(x_{i}(t)\right), f_{2}\left(x_{i}(t)\right), \ldots, f_{n}\left(x_{i}(t)\right)\right)^{T}$ in network (1) satisfies the local Lipschitz condition, for any compact set $\mathbb{S} \in R^{m}$, there exists a positive constant $\eta(\mathbb{S})$, such that

$$
(x-y)^{T}[f(x)-f(y)] \leq \eta(\mathbb{S})\|x-y\|^{2}, \quad \forall x, y \in \mathbb{S} .
$$


Assumption 2. The synchronous state $x_{0}$ is bounded; that is, there exists a compact set $\mathbb{S}=\mathbb{S}\left(x_{0}(0)\right) \in R^{n}$ such that the trajectory of (3) starting from $x_{0}(0)$ is always in the compact set $\mathbb{S}$.

Remark 4. Note that the nonlinear dynamics of network (1) only satisfies the local Lipschitz condition. If the Jacobian matrix of $f$ is continuous, then $f$ is at least local Lipschitz. Many famous systems may not be governed by global Lipschitz nonlinearity but by local Lipschitz nonlinearity, such as Lorenz system and Chen system; therefore, it is worthy of discussing the local Lipschitz nonlinearity dynamics.

Assumption 3 (see [13]). The interconnection graph $\mathscr{G}$ remains connected.

\subsubsection{Lemmas}

Lemma 5 (see [2]). If $A=\left(a_{i j}\right) \in R^{N \times N}$ is a symmetric irreducible matrix with $a_{i i}=-\sum_{j=1, j \neq i}^{N} a_{i j}$ and the matrix $E=\operatorname{diag}(e, 0, \ldots, 0)$ with $e>0$, then all eigenvalues of the matrix $A-E$ are negative.

Lemma 6 (see [18], Chain Rule). Let $x(\cdot)$ be a Filippov solution to $\dot{x}=f(x, t)$ on an interval containing $t$ and let $V: R^{n} \times R \rightarrow R$ be a Lipschitz and regular function. Then $V(x(t), t)$ is absolutely continuous, $(d / d t) V(x(t), t)$ exists almost everywhere, and

$$
\frac{d}{d t} V(x(t), t) \stackrel{\text { a.e. }}{\in} \dot{\widetilde{V}}(x, t)
$$

where $\dot{\widetilde{V}}(x, t):=\bigcap_{\xi \in \partial V(x(t), t)} \xi^{T}\left[\begin{array}{c}K f(x(t), t) \\ 1\end{array}\right]$.

Remark 7 (see [12]). In Lemma 6, the (global) Lipschitz continuity required to $V(x)$ can be relaxed to the local condition.

Lemma 8 (see [17], Lyapunov's theorem generalized). If

(i) $V: R^{n} \rightarrow R, V(0)=0$ and $V(x)>0$, for all $x \neq 0$;

(ii) $x: R \rightarrow R^{n}$ and $V(x(t))$ is absolutely continuous on $[0,+\infty)$ with

$$
\frac{d}{d t}[V(x(t))]<-\varepsilon<0 \quad \text { a.e. on }\{t \mid x(t) \neq 0\},
$$

then $x$ converges to 0 in finite time.

It is worth noting that if the condition $V(0)=0$ in (i) of Lemma 8 cannot hold; then we can have the following lemma.

Lemma 9. If

(i) $V: R^{n} \rightarrow R, V(x)>0$, for all $x \neq 0$;

(ii) $x: R \rightarrow R^{n}$ and $V(x(t))$ is absolutely continuous on $[0,+\infty)$ with

$$
\frac{d}{d t}[V(x(t))]<-\varepsilon<0 \quad \text { a.e. on }\{t \mid x(t) \neq 0\} \text {, }
$$

then $x$ converges to 0 as $t \rightarrow \infty$.
Proof. If Lemma 9 is not true, then we have $x(t) \neq 0$, for all $t \epsilon$ $\left[t_{0},+\infty\right)$ and $(d / d t)[V(x(t))]<-\varepsilon<0$ a.e. on $\{t \mid x(t) \neq 0\}$. Therefore,

$$
\lim _{t \rightarrow \infty} V(t)=V(x(0))+\int_{0}^{\infty} \dot{V}(t) d t=-\infty,
$$

since $V(x(0))$ is bounded, which contradicts with $V(x)>0$, for all $x \neq 0$.

This completes the proof.

\section{Main Results}

Denote

$$
d_{0} \triangleq \frac{1}{2} \sum_{i=1}^{N}\left(x_{i}(0)-x_{0}\right)^{2}
$$

and construct a closed space

$$
\mathbb{B}\left(\sigma d_{0}, x_{0}\right)=\left\{x \in R^{N n} \mid \frac{1}{2} \sum_{i=1}^{N}\left\|x_{i}-x_{0}\right\|^{2} \leq \sigma d_{0}\right\},
$$

where $x=\left(x_{1}^{T}, x_{2}^{T}, \ldots, x_{N}^{T}\right)^{T}$ and $\sigma>1$ is a constant. Under Assumption 1 , for all $x \in \mathbb{B}\left(\sigma d_{0}, x_{0}\right)$, there exists a constant $\eta\left(\sigma, x_{0}\right)$ such that

$$
\sum_{i=1}^{N}\left(x_{i}-x_{0}\right)^{T}\left(f\left(x_{i}\right)-f\left(x_{0}\right)\right) \leq \eta\left(\sigma, x_{0}\right)\left\|x_{i}-x_{0}\right\|^{2} .
$$

In order to get the main result, we construct the following potential function:

$$
\begin{aligned}
V(t)= & \frac{1}{2} \sum_{i=1}^{N} \tilde{x}_{i}^{T}(t) \tilde{x}_{i}(t)+\sum_{i=1}^{N} \sum_{j \in N_{i}(t)} \frac{\left(c_{i j}(t)-m\right)^{2}}{4 k_{i j}} \\
& +\sum_{i=1}^{N} \frac{\left(c_{i}(t)-m\right)^{2}}{2 k_{i}},
\end{aligned}
$$

where $m>0$ is sufficiently large. For convenience, we denote $c=\left(c_{i j}\right)_{N \times N}$ and $\widetilde{c}=\left(c_{1}^{T}, c_{2}^{T}, \ldots, c_{N}^{T}\right)^{T}$. Then, we can have the following results.

Theorem 10. Supposing that Assumptions 1-3 hold, then the combined trajectories of all nodes and the parameters $(x, c, \widetilde{c})$ in (1) and (2) are geared to a compact hyper-ellipsoid

$$
\Omega\left(\sigma d_{0}, m, x_{0}\right)=\left\{(x, c, \widetilde{c}) \in R^{N n} \times R^{N n} \times R^{N} \mid V<\sigma d_{0}\right\},
$$

if the initial value is selected from

$$
\begin{aligned}
& \Omega_{0}\left(\sigma d_{0}, m, x_{0}\right) \\
& =\left\{(x, c, \widetilde{c}) \in R^{N n} \times R^{N n} \times R^{N} \mid V(0)<\sigma d_{0}\right\} .
\end{aligned}
$$


Proof. Taking the derivative of $V(t)$ in $\left[t_{0}, t_{1}\right)$, we can get

$$
\begin{aligned}
\dot{V}(t)= & \sum_{i=1}^{N} \tilde{x}_{i}^{T}(t)\left[f\left(x_{i}(t)\right)-f\left(x_{0}(t)\right)\right. \\
& \left.+\sum_{j \in N_{i}} a_{i j} c_{i j}(t)\left(\tilde{x}_{j}(t)-\tilde{x}_{i}(t)\right)\right] \\
& +\frac{1}{2} \sum_{i=1}^{N} \sum_{j \in N_{i}}\left(c_{i j}(t)-m\right) a_{i j}\left(\tilde{x}_{i}(t)-\tilde{x}_{j}(t)\right)^{T} \\
& \times \sum_{i=1}^{N}\left(c_{i}(t)-m\right) h_{i} \tilde{x}_{i}^{T}(t) \tilde{x}_{i}(t) \\
= & \sum_{i=1}^{N} \tilde{x}_{i}^{T}(t)\left(\eta\left(\sigma, \tilde{x}_{0}\right) I_{N}\right) \tilde{x}_{i}(t) \\
& -\frac{1}{2} m \sum_{i=1}^{N} \sum_{j \in N_{i}} a_{i j}\left(\tilde{x}_{i}(t)-\tilde{x}_{j}(t)\right)^{T}\left(\tilde{x}_{i}(t)-\tilde{x}_{j}(t)\right) \\
& -\tilde{x}^{T}(t)\left[\left(\left(\eta\left(\sigma, x_{0}\right) I_{N}\right)+m(A-H)\right) \otimes I_{n}\right] \tilde{x}(t) .
\end{aligned}
$$

Under Assumption 3, Lemma 5 implies that $A-H<0$ in $\left[t_{0}, t_{1}\right)$. Since $m$ is a sufficiently large positive constant, then

$$
\dot{V} \leq 0
$$

which implies

$$
V(t) \leq V(0), \quad t \in\left[t_{0}, t_{1}\right)
$$

Similarly,

$$
V(t) \leq V\left(t_{k-1}\right), \quad t \in\left[t_{k-1}, t_{k}\right), k=1,2, \ldots, m .
$$

Thus, we have

$$
V(t) \leq V(0)<\sigma d_{0} .
$$

Therefore, we can conclude that the combined trajectories of all nodes with the parameters $(x, c, \widetilde{c})$ are geared to a compact hyper-ellipsoid $\Omega\left(\sigma d_{0}, m, x_{0}\right)=\left\{(x, c, \widetilde{c}) \in R^{N n} \times R^{N n} \times R^{N}\right.$ | $\left.V<\sigma d_{0}\right\}$ if the initial value is selected from $\Omega_{0}\left(\sigma d_{0}, m, x_{0}\right)=$ $\left\{(x, c, \widetilde{c}) \in R^{N n} \times R^{N n} \times R^{N} \mid V(0)<\sigma d_{0}\right\}$.

This completes the proof.

Theorem 11. If network (1) steered by adaptive laws (3) and the initial value of $(x, c, \widetilde{c})$ is defined as (18), under Assumptions 13 , then all nodes will converge to the synchronous state even when only one node is controlled by (2).

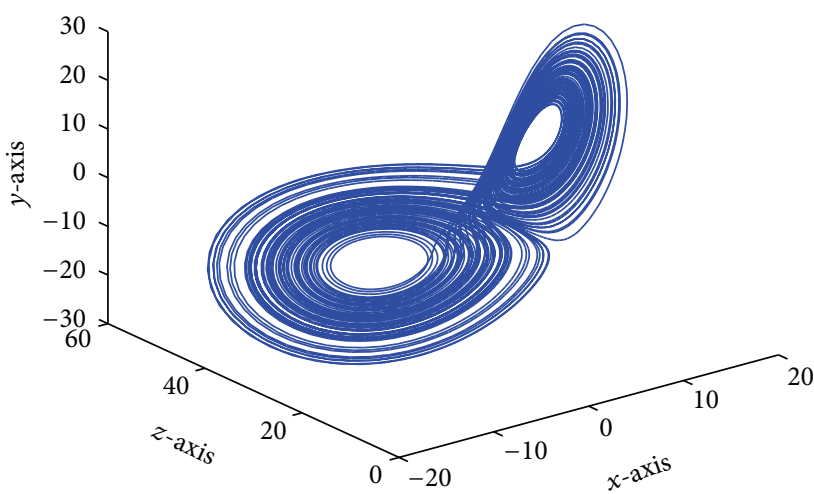

FIGURE 1: Trajectories of the synchronous state.

Proof. Let $\tilde{x}_{i}(t)=x_{i}(t)-\bar{x}(t)$. Construct the Lyapunov function, one has

$$
\begin{aligned}
V(t)= & \frac{1}{2} \sum_{i=1}^{N} \tilde{x}_{i}^{T}(t) \tilde{x}_{i}(t)+\sum_{i=1}^{N} \sum_{j \in N_{i}(t)} \frac{\left(c_{i j}(t)-m\right)^{2}}{4 k_{i j}} \\
& +\sum_{i=1}^{N} \frac{\left(c_{i}(t)-m\right)^{2}}{2 k_{i}},
\end{aligned}
$$

where $m>0$ is a sufficiently large constant.

By Definition 1, we can obtain

$$
\begin{aligned}
& \dot{x}_{i}(t) \stackrel{\text { a.e. }}{\epsilon} K\left[f\left(x_{i}(t)\right)+\sum_{j \in N_{i}(t)} a_{i j} c_{i j}(t)\left(x_{j}(t)-x_{i}(t)\right)\right. \\
&\left.-h_{i} c_{i}(t)\left(x_{i}(t)-\bar{x}(t)\right)\right], \\
& \dot{c}_{i j}(t) \stackrel{\text { a.e. }}{\epsilon} K\left[k_{i j} a_{i j}\left(x_{i}(t)-x_{j}(t)\right)^{T}\left(x_{i}(t)-x_{j}(t)\right)\right] .
\end{aligned}
$$

Because the topology of network (1) is switching, we have

$$
\begin{aligned}
\dot{x}_{i}(t) & \subseteq K\left[f\left(x_{i}(t)\right)+\sum_{j \in N_{i}(t)} a_{i j} c_{i j}(t)\left(x_{j}(t)-x_{i}(t)\right)\right] \\
& -h_{i} c_{i}(t)\left(x_{i}(t)-\bar{x}(t)\right) .
\end{aligned}
$$

Also by Definition 3, we know that $V(t)$ is regular, then

$$
\begin{aligned}
\partial V \subseteq[ & \tilde{x}_{1}^{T}(t), \ldots, \tilde{x}_{N}^{T}(t), \sum_{j=2}^{N} \frac{\left(c_{1 j}(t)-m\right)}{2 k_{1 j}}, \ldots, \\
& \left.\sum_{j=1}^{N-1} \frac{\left(c_{N-1 j}(t)-m\right)}{2 k_{N-1 j}}, \frac{c_{1}(t)-m}{k_{1}}, \ldots, \frac{c_{N}(t)-m}{k_{N}}\right]^{T} .
\end{aligned}
$$




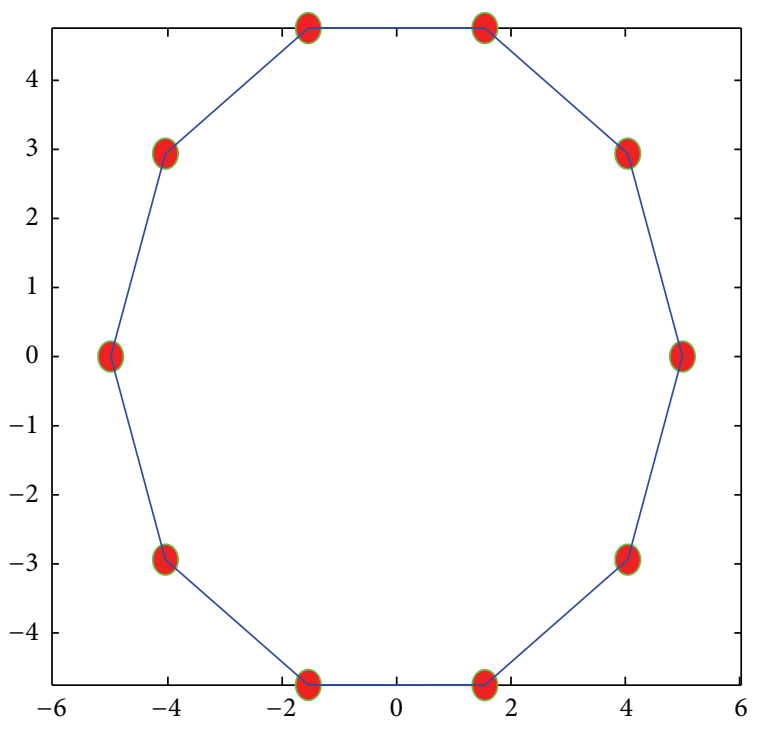

(a)

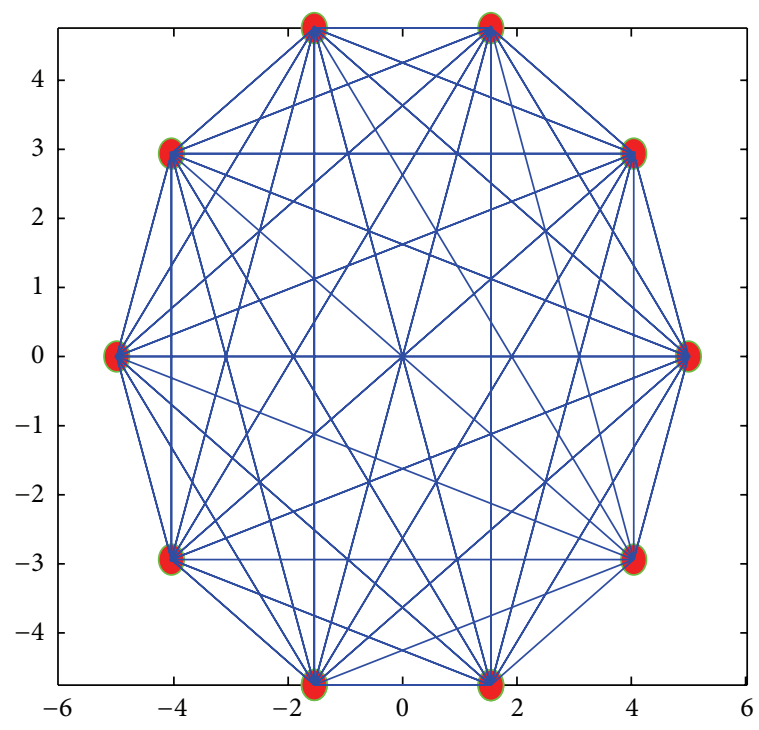

(b)

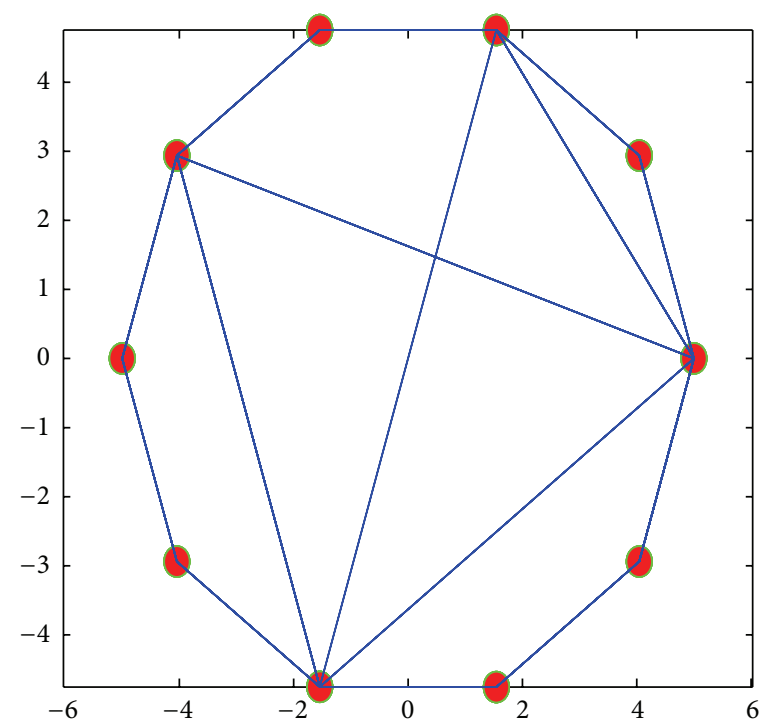

(c)

FIGURE 2: The topologies of network (1).

Hence,

$$
\begin{aligned}
\dot{\tilde{V}} \subset K\left[\sum _ { i = 1 } ^ { N } \tilde { x } _ { i } ^ { T } ( t ) \left[f\left(x_{i}(t)\right)-f\left(x_{0}(t)\right)\right.\right. \\
\left.\left.+\sum_{j \in N_{i}} a_{i j} c_{i j}(t)\left(\tilde{x}_{j}(t)-\tilde{x}_{i}(t)\right)\right]\right] \\
+K\left[\frac{1}{2} \sum_{i=1}^{N} \sum_{j \in N_{i}}\left(c_{i j}(t)-m\right) a_{i j}\left(\tilde{x}_{i}(t)-\tilde{x}_{j}(t)\right)^{T}\right. \\
\left.\quad \times\left(\tilde{x}_{i}(t)-\tilde{x}_{j}(t)\right)+\sum_{i=1}^{N}\left(c_{i}(t)-m\right) h_{i} \tilde{x}_{i}^{T}(t) \tilde{x}_{i}(t)\right]
\end{aligned}
$$

Consequently,

$\dot{\tilde{V}} \subset K\left[\widetilde{x}^{T}(t)\left[\left(\left(\eta\left(\sigma, x_{0}\right) I_{N}\right)+m(A-H)\right) \otimes I_{n}\right] \tilde{x}(t)\right]$. 

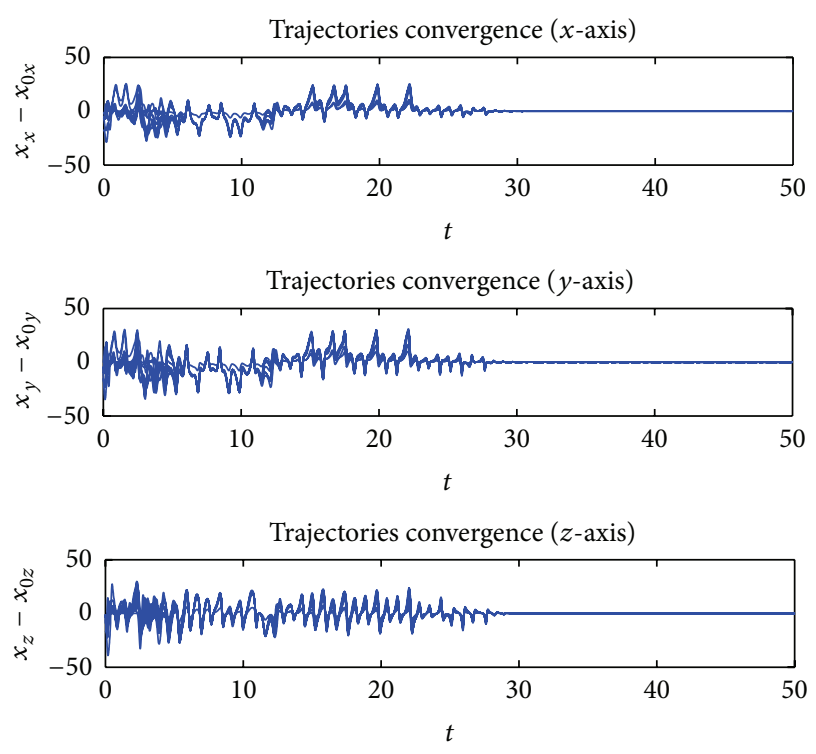

FIGURE 3: Trajectories convergence of 10 nodes with only one node informed by controllers (2).

Lemma 5 indicates that all the eigenvalues of $A-H$ are negative, and there exists $\varepsilon>0$ such that

$$
\frac{d}{d t}[V(x(t))]<-\varepsilon<0 \quad \text { a.e. on }\{t \mid x(t) \neq 0\},
$$

since $m$ is a sufficiently large positive constant.

From Lemma 9, we can get that all the nodes of network (1) can converge to the synchronous state $\bar{x}(t)$. This completes the proof.

Remark 12. Note also that Theorem 11 is still established when $A^{k}$ is asymmetric.

Similar to the proof of Theorem 11, we also have

$$
\begin{aligned}
\dot{\widetilde{V}} \subset K\left[\tilde{x}^{T}(t)[(\right. & \left(\eta\left(\sigma, x_{0}\right) I_{N}\right) \\
& \left.\left.\left.+m\left(\frac{A+A^{T}}{2}-H\right)\right) \otimes I_{n}\right] \tilde{x}(t)\right],
\end{aligned}
$$

since $\left(A+A^{T}\right) / 2$ is symmetric.

\section{Simulations}

In this section, number simulations are given to illustrate our theoretical results. All nodes of network (1) and the synchronous goal share the same nonlinear dynamics described as the Lorenz system as follows:

$$
f(x(t))=f\left(x^{1}, x^{2}, x^{3}\right)=\left\{\begin{array}{l}
\dot{x}^{1}=10\left(x^{2}-x^{1}\right), \\
\dot{x}^{2}=28 x^{1}-x^{1} x^{3}-x^{2}, \\
\dot{x}^{3}=x^{1} x^{2}-\frac{8}{3} x^{3},
\end{array}\right.
$$

as shown in Figure 1. Then the network can be shown as follows:

$$
\begin{aligned}
{\left[\begin{array}{c}
\dot{x}_{i}^{1} \\
\dot{x}_{i}^{2} \\
\dot{x}_{i}^{3}
\end{array}\right]=\left[\begin{array}{c}
10\left(x_{i}^{2}-x_{i}^{1}\right) \\
28 x_{i}^{1}-x_{i}^{1} x_{i}^{3}-x_{i}^{2} \\
x_{i}^{1} x_{i}^{2}-\frac{8}{3} x_{i}^{3}
\end{array}\right] } \\
+\sum_{j \in N_{i}(t)} a_{i j}^{\sigma(t)} c_{i j}^{\sigma(t)}(t)\left(x_{j}(t)-x_{i}(t)\right) \\
-h_{i} c_{i}(t)\left(x_{i}(t)-x_{0}(t)\right) .
\end{aligned}
$$

Figure 2 shows the topology of network (1) with subgraphs (a), (b), and (c) during the time intervals $[0,4],[4,12]$, $[12,50]$, respectively.

The initial values of the 10 nodes are chosen randomly, and the initial value of the synchronous goal is $x_{0}(0)=$ $[10,10,10]^{T}$. There is only one node informed by the synchronous goal, and the 10th node is given, that is, $h_{i}(i=$ $1,2, \ldots, 9)=0$ and $h_{10}=1$. The initial values of the adaptive parameters are $c_{i j}(0)=0$ with the weight of $k_{i j}(0)=0$ for all $i$ and $j$, while $c_{i}(0)=0$ with the weight of $k_{i}(0)=0$ for all $i$.

Figure 3 describes the convergence of the state errors on the $x$-axis, $y$-axis, and $z$-axis, respectively. From this figure, we can see that all nodes of network (1) can synchronize to the synchronous state when the neighboring graph $\mathbb{G}$ remains connected with switching topology even though only one node is informed by the synchronous state. Figure 4 shows the change trends of the adaptive coupling strengths and the adaptive feedback gains, respectively, and all these parameters converge to the constants.

\section{Conclusion}

In this paper, we have investigated the synchronization of complex dynamical networks with switching topology via differential inclusion method. Different from the most previous work, all nodes and the synchronous state in this paper share the same intrinsic nonlinear dynamics governed by the local Lispchitz condition. By adding decentralized adaptive strategies to the coupling strengths and the feedback gains, all nodes can converge to the synchronous state even when only one node is pinning controlled by the synchronous state if the neighboring graph $\mathscr{G}$ of the switching topology remains connected.

\section{Acknowledgments}

This work was supported in part by the National Natural Science Foundation of China under Grants (61304049, 61104140, 61203150, 61170113, 61174116), Beijing Natural Science Foundation Program 4122019, Funding Project for Academic Human Resources Development in Institutions of Higher Learning under the jurisdiction of Beijing Municipality (PHR201108055), Science and Technology Development Plan Project of Beijing Education Commission (no. KM201310009011), the Fundamental Research Funds for the Central Universities (HUST: Grant no. 2011JC055), the 


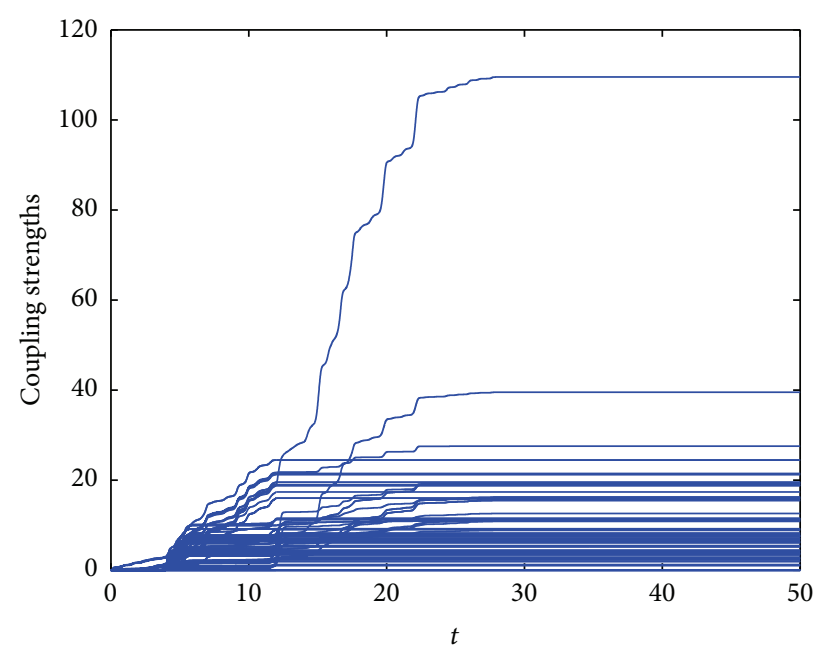

(a) Coupling strengths

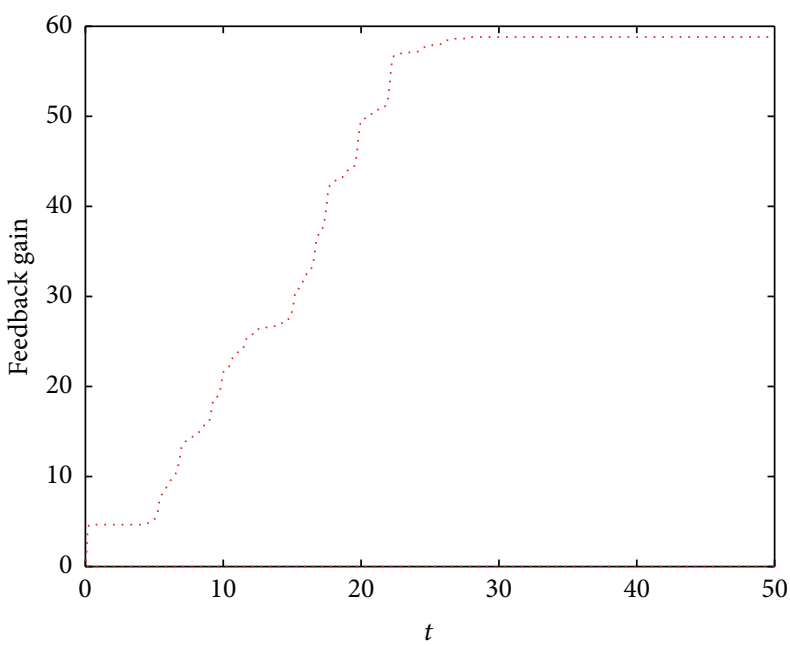

(b) Feedback gain

FIGURE 4: Convergence of the adaptive coupling strengths and the feedback gain.

Research Fund for the Doctoral Program of Higher Education (RFDP) under Grant no. 20100142120023, and the Natural Science Foundation of Hubei Province of China under Grant no. 2011CDB042.

\section{References}

[1] B. Liu, X. Wang, H. Su, Y. Gao, and L. Wang, "Adaptive secondorder consensus of multi-agent systems with heterogeneous nonlinear dynamics and time-varying delays," Neurocomputing, vol. 118, pp. 289-300, 2013.

[2] H. Su, X. Wang, and Z. Lin, "Flocking of multi-agents with a virtual leader," IEEE Transactions on Automatic Control, vol. 54, no. 2, pp. 293-307, 2009.

[3] H. Su, Z. Rong, M. Z. Q. Chen, X. Wang, G. Chen, and H. Wang, "Decentralized adaptive pinning control for cluster synchronization of complex dynamical networks," IEEE Transactions on Cybernetics, vol. 43, no. 1, pp. 394-399, 2013.

[4] H. Su, G. Chen, X. Wang, and Z. Lin, "Adaptive secondorder consensus of networked mobile agents with nonlinear dynamics," Automatica, vol. 47, no. 2, pp. 368-375, 2011.

[5] D. J. Stilwell, E. M. Bollt, and D. G. Roberson, "Sufficient conditions for fast switching synchronization in time-varying network topologies," SIAM Journal on Applied Dynamical Systems, vol. 5, no. 1, pp. 140-156, 2006.

[6] J. Zhao, D. J. Hill, and T. Liu, "Synchronization of complex dynamical networks with switching topology: a switched system point of view," Automatica, vol. 45, no. 11, pp. 2502-2511, 2009.

[7] P. Lin and Y. Jia, "Average consensus in networks of multi-agents with both switching topology and coupling time-delay," Physica A, vol. 387, no. 1, pp. 303-313, 2008.

[8] J. Yao, D. J. Hill, Z.-H. Guan, and H. O. Wang, "Synchronization of complex dynamical networks with switching topology via adaptive control," in Proceedings of the 45th IEEE Conference on Decision and Control (CDC '06), pp. 2819-2824, San Diego, Calif, USA, December 2006.
[9] W. Lin, "Adaptive chaos control and synchronization in only locally Lipschitz systems," Physics Letters A, vol. 372, no. 18, pp. 3195-3200, 2008.

[10] Y. Hu, H. Su, and J. Lam, "Adaptive consensus with a virtual leader of multiple agents governed by locally Lipschitz nonlinearity," International Journal of Robust and Nonlinear Control, vol. 23, no. 9, pp. 978-990, 2013.

[11] H. Su, N. Zhang, M. Chen, H. Wang, and X. Wang, "Adaptive flocking with a virtual leader of multiple agents governed by local Lipschitz nonlinearity," Nonlinear Analysis: Real World Applications, vol. 14, no. 1, pp. 798-806, 2012.

[12] H. G. Tanner, A. Jadbabaie, and G. J. Pappas, "Stable flocking of mobile agents part II: dynamic topology," in Proceedings of the 42nd IEEE Conference on Decision and Control (CDC '03), pp. 2016-2021, December 2003.

[13] H. Shi, L. Wang, T. Chu, and M. Xu, "Flocking coordination of multiple mobile autonomous agents with asymmetric interactions and switching topology," in Proceedings of the IEEE IRS/RSJ International Conference on Intelligent Robots and Systems (IROS '05), pp. 935-940, August 2005.

[14] H. Shi, L. Wang, T. Chu, G. Xie, and M. Xu, "Flocking coordination of multiple interactive dynamical agents with switching topology," in Proceedings of the IEEE International Conference on Systems, Man and Cybernetics, pp. 2684-2689, October 2006.

[15] H. Shi, L. Wang, T. Chu, and M. Xu, "Flocking control of multiple interactive dynamical agents with switching topology via local feedback," in Advances in Artificial Life, vol. 3630 of Lecture Notes in Computer Science, pp. 604-613, Springer, Berlin, Germany, 2005.

[16] F. H. Clarke, Yu. S. Ledyaev, R. J. Stern, and P. R. Wolenski, Nonsmooth Analysis and Control Theory, vol. 178 of Graduate Texts in Mathematics, Springer, New York, NY, USA, 1998.

[17] B. E. Paden and S. S. Sastry, "A calculus for computing Filippov's differential inclusion with application to the variable structure control of robot manipulators," IEEE Transactions on Circuits and Systems, vol. 34, no. 1, pp. 73-82, 1987.

[18] D. Shevitz and B. Paden, "Lyapunov stability theorey of nonsmooth systems," in Proceeding of the 32nd Conference on Decision and Control, 1993. 


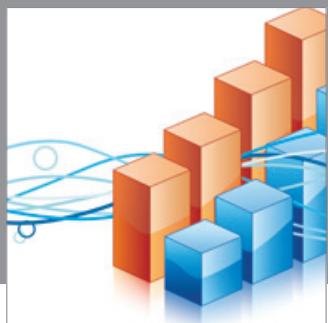

Advances in

Operations Research

mansans

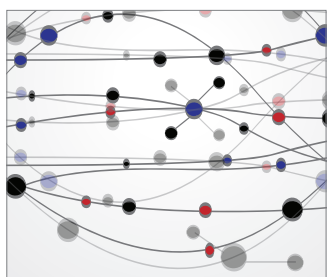

The Scientific World Journal
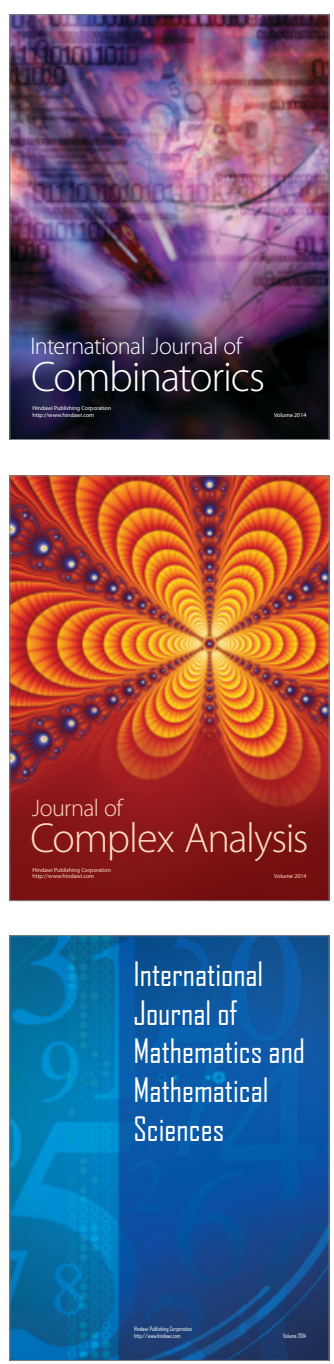
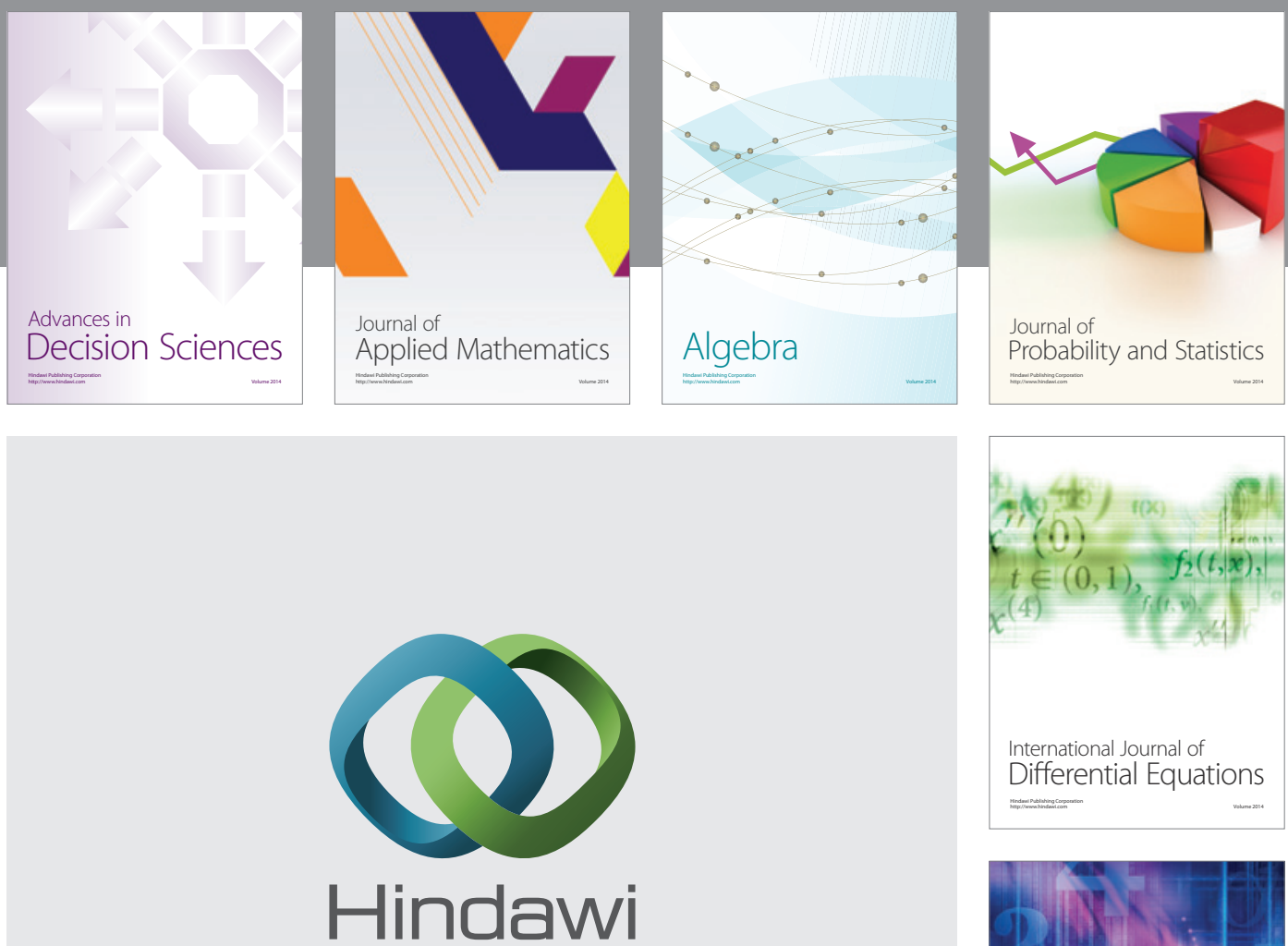

Submit your manuscripts at http://www.hindawi.com
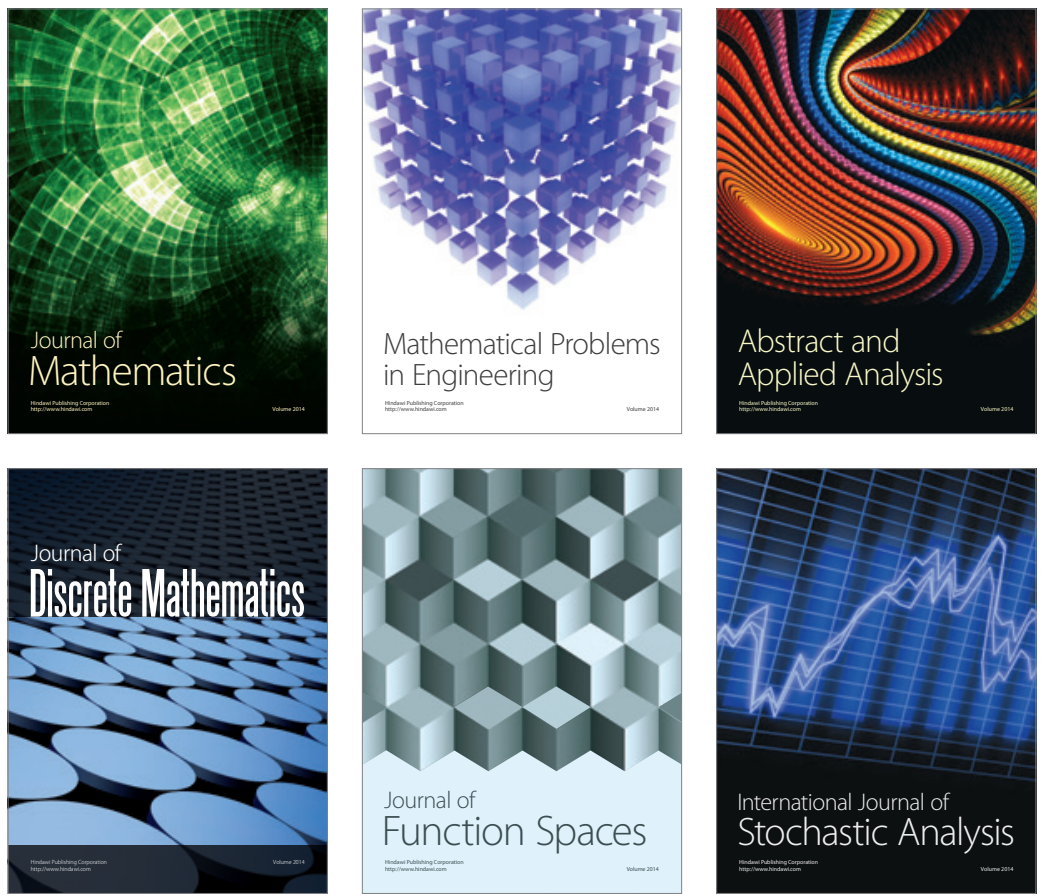

Journal of

Function Spaces

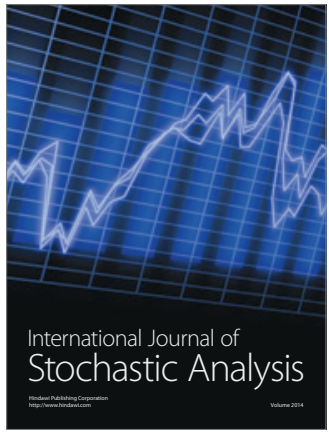

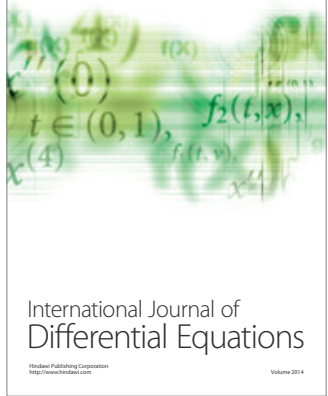
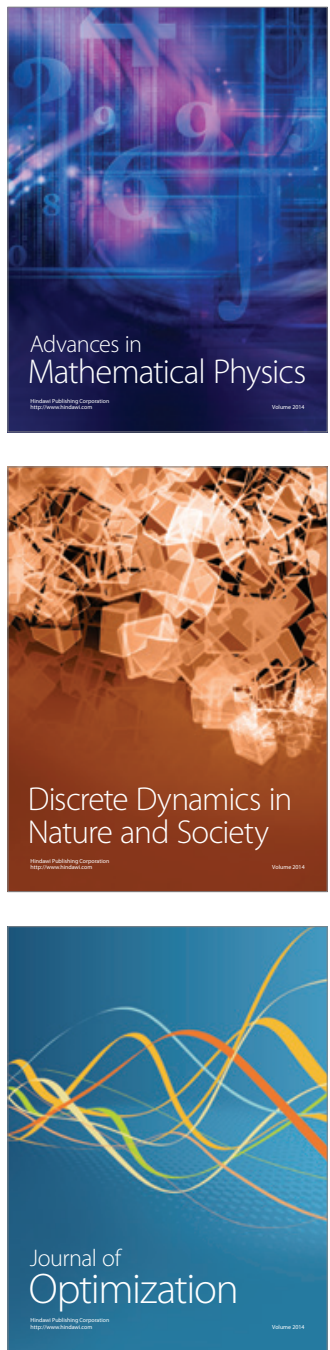